\title{
Radón en la mina Coricancha, San Mateo de Huánchor
}

\author{
José Manuel Osores ${ }^{1}$, Susana Gonzáles ${ }^{1}$, Jorge Martínez ${ }^{1}$, Raúl Jara ${ }^{1}$, Vicente de Paulo Melo² \\ 1 Instituto Peruano de Energía Nuclear, Av. Canadá 1470, Lima 41, Perú \\ 2 Instituto de Radioproteção e Dosimetria, Av. Salvador Allende 9, B. de Tijuca, Río de Janeiro, Brasil
}

Recibido el 15 de junio 2018. Aceptado 21 de junio 2018

DOI: https://doi.org/10.33017/RevECIPeru2018.0002/

\section{Resumen}

Se realizó la monitorización de radón en la mina Coricancha con la finalidad de establecer una línea base sobre los niveles de concentración de este radionucleído en una mina subterránea de la región andina del país. Las concentraciones obtenidas se encuentran distribuidas en un amplio rango, desde los 245 a $1897 \mathrm{~Bq} / \mathrm{m}^{3}$, con una media de $565 \mathrm{~Bq} / \mathrm{m}^{3}$, valor que se encuentra por debajo del límite establecido en el Reglamento de Seguridad Radiológica.

Descriptores: Radón, mina subterránea, NORM.

\section{Abstract}

Radon monitoring was carried out at the Coricancha mine in order to establish a baseline on the concentration levels of this radionuclide in an underground mine in the Andean region of the country. The concentrations obtained are distributed in a wide range, from 245 to $1897 \mathrm{~Bq} / \mathrm{m}^{3}$, with an average of $565 \mathrm{~Bq} / \mathrm{m}^{3}$, a value that is below the limit established in the Regulation of Radiological Safety.

Keywords: Radon, underground mine, NORM.

\section{Introducción}

El radón es un gas natural radiactivo que se produce por la desintegración del uranio que se encuentra presente en la corteza terrestre. Constituye uno de los principales materiales radiactivos de origen natural (NORM). Este radionucleído decae en otros elementos radiactivos los cuales al ser inhalados penetran en las vías respiratorias dañando a las células con la radiación emitida [1].

El radón fue identificado como un problema de salud cuando los científicos se dieron cuenta que los mineros de uranio trabajando bajo tierra que estaban expuestos al radón morían por cáncer de pulmón en índices superiores a lo que se esperaba [2].

La mayor parte de lo que se conoce acerca del radón como causante potencial de cáncer proviene del estudio sobre estos mineros los cuales fueron luego confirmados por estudios con animales, que muestran índices más altos de tumores en el pulmón en los roedores expuestos a altas cantidades de radón.

El proceso degenerativo del gas radón crea productos nocivos para el ser humano. Algunas partículas pueden llegar a los pulmones con aire, humo, o polvo e irradiar tejidos sanos. Esto puede llevar a la formación de células cancerosas, y explica porque los mineros que fuman son veinte veces más propensos a sufrir cáncer pulmonar que los no fumadores.

Los primeros antecedentes en el país sobre radón fueron los obtenidos durante la prospección de uranio en el territorio nacional, en estos trabajos se detectaron varias anomalías de radón asociadas tanto a la mineralización del uranio, así como su relación en ambientes de rocas graníticas y volcánicas, en algunas minas polimetálicas y 
fuentes a aguas termales [3]. En la actualidad existen dos tipos de pruebas para detectar el radón: de corta y de larga duración.

Las pruebas de corta duración son las más rápidas; estas pruebas cortas permanecen en el ambiente de dos a noventa días, según el tipo de dispositivo que se utilice. Entre los detectores más usados en las pruebas de corta duración se tienen las "latas de carbón" (charcoal canisters), "bolsas de carbón" (charcoal bags), "detectores de partículas alfa" (alpha track detectors), "cámara de iones eléctricos" (electret ion chambers), "monitores continuos" (continuous monitors), y "centelleo de líquido de carbón" (charcoal liquid scintillation) [4].

En el Perú no se conoce la magnitud del impacto de las concentraciones de radón en minas subterráneas; al no conocerse los niveles del gas radón y sus productos de decaimiento en minas subterráneas, no es posible desarrollar estudios radio-epidemiológicos en los trabajadores mineros y por lo tanto no es posible evaluar el impacto ambiental sobre esta población ocupacionalmente expuesta ni establecer programas de gestión ambiental para esta actividad.

En este sentido, existe la necesidad de establecer y estandarizar procedimientos nuevos para una adecuada monitorización de radón en nuestro país, teniendo en cuenta nuestra capacidad nacional instalada, los costos operativos y las facilidades de muestreo en el territorio nacional con la finalidad de proponer instrumentos normativos (leyes y reglamentos) para garantizar la calidad de vida de nuestra población.

El proyecto "Estudio piloto para la determinación de los niveles de radón en minas subterráneas", tiene por objetivo general, desarrollar una nueva metodología para monitorizar los niveles de emisión de gas radón en minas subterráneas a través de la evaluación de las diferentes metodologías utilizadas para la determinación de este radionucleído y sus productos de decaimiento.

\section{Metodología}

\subsection{Lugar de ejecución}

La presente investigación se llevó a cabo tomando como punto de muestreo la mina Coricancha, la cual se encuentra ubicada en el Distrito de San Mateo, provincia de Huarochirí, Lima, Perú, en los $11^{\circ} 47^{\prime} 82^{\prime \prime}$ de Latitud Sur, $76^{\circ} 17^{\prime} 83^{\prime \prime}$ de Longitud
Oeste y a 3700 metros sobre el nivel del mar (Figura 1).

\subsection{Evaluación de la zona de estudio}

Se llevó a cabo la evaluación integral de la zona donde se ejecutó la investigación a fin de recopilar información sobre las características de la mina, su capacidad productiva, ubicación geográfica y altitud, así como la determinación del lugar más conveniente para instalar los dispositivos de monitoreo de radón en aire.

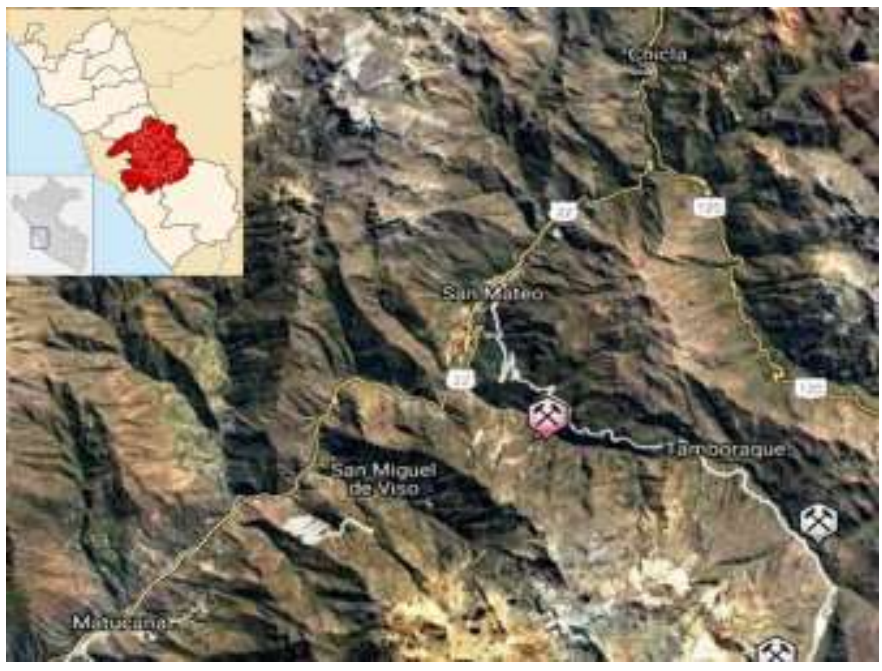

Figura 1: Ubicación de la mina Coricancha.

\subsection{Instalación de dispositivos para radón}

Una vez seleccionada la mina y ubicación de los dispositivos, se instaló un total de diez dispositivos facilitados por el Instituto de Radioprotección y Dosimetría de Brasil (IRD), colocándolos en un soporte a un metro de distancia de las paredes de la mina y un metro de altura (Figura 2).

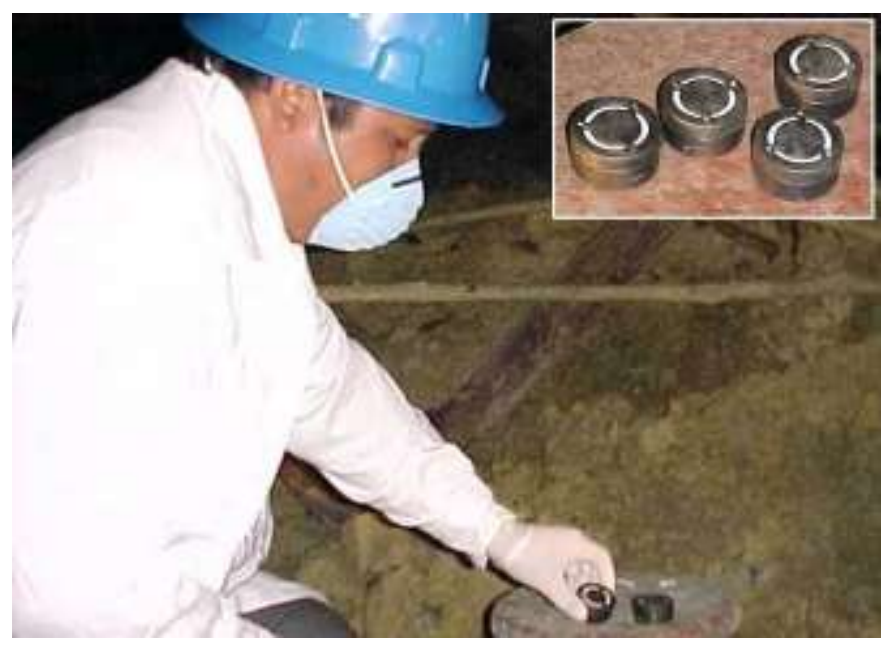

Figura 2: Instalación de dispositivos para radón. 
Estos dispositivos fueron retirados por duplicado cada semana cubriendo un total de cinco semanas de monitorización, de modo tal que se puede estimar el tiempo óptimo de monitorización y el tiempo requerido para la saturación de los dispositivos. Los dispositivos colectados fueron colocados dentro de una envoltura de plástico de 2 micras de espesor la cual fue sellada para evitar el ingreso de radón de otras áreas, luego, esta envoltura se colocó dentro de una envoltura de aluminio plastificado procediéndose a sellarla para remitirla a los laboratorios de IPEN y luego enviarlas al IRD-Brasil por vía postal.

\subsection{Instalación de dosímetros TLD}

Como ensayo complementario para evaluar la dosis ambiental en el interior de la mina, se instalaron dosímetros termoluminiscentes (TLD) compuestos de fluoruro de calcio activado con disprosio (CaF2: Dy). Estos dosímetros fueron colocados durante un mes en el interior de la mina al cabo del cual fue retirado y remitido al Laboratorio Secundario de Calibraciones Dosimétricas (LSCD) del IPEN para las evaluaciones correspondientes.

\section{Resultados}

\subsection{Evaluación de la zona de estudio}

Las operaciones de Tamboraque se encuentran en el km 90 de la Carretera Central Lima-La Oroya, se ubican en el distrito de San Mateo de Huanchor, provincia de Huarochirí, departamento de Lima a una elevación de $\mathbf{2 8 9 0}$ metros sobre el nivel del mar.

La mina Coricancha está conformada por un yacimiento polimetálico de vetas, emplazadas en volcánicos andesíticos terciarios. Está caracterizada por ser un yacimiento de vetas polimetálicas, de relleno hidrotermal dentro de zonas de fracturamiento, cizallamiento y localmente zonas tensionales. Las vetas tienen una potencia que varía de 0,30 a 0,80 metros, presentando ensanchamientos locales y sistemas de lazos sigmoides.

La mineralización está formada por arsenopirita, pirita, esfalerita ferrífera, galena argentífera, calcopirita y cuarzo. El oro está directamente relacionado con la arsenopirita y la pirita, la plata y el plomo con la galena y el zinc con la esfalerita.

Durante la época del desarrollo experimental del proyecto, se registró una temperatura promedio de $26,8{ }^{\circ} \mathrm{C}$ en el interior de la mina y una humedad relativa del $58 \%$.

\subsection{Concentración de radón en la mina}

En la Tabla 1 se observa un amplio rango de variabilidad en las concentraciones de radón durante el periodo de muestreo, con un promedio de $874,6 \pm 556,9 \mathrm{~Bq} / \mathrm{m}^{3}$.

Tabla 1: Concentración de radón en la mina Coricancha.

\begin{tabular}{|c|c|c|c|c|}
\hline $\begin{array}{c}\text { Tiempo de } \\
\text { Exposició } \\
\mathbf{n} \\
\text { (días) }\end{array}$ & $\begin{array}{c}\text { Trazas } \\
\text { Totales }\end{array}$ & $\begin{array}{c}\text { Trazas } \\
\text { Netas }\end{array}$ & $\begin{array}{c}\text { Concentració } \\
\mathbf{n} \\
\mathbf{( B q} / \mathbf{m} 3)\end{array}$ & $\begin{array}{c}\text { Desviació } \\
\mathbf{n} \\
\mathbf{( B q} / \mathbf{m} 3)\end{array}$ \\
\hline 7,01 & $\begin{array}{c}195,1 \\
5\end{array}$ & $\begin{array}{c}183,6 \\
5\end{array}$ & 1897,37 & 144,33 \\
\hline 7,01 & $\begin{array}{c}134,8 \\
0\end{array}$ & $\begin{array}{c}123,3 \\
0\end{array}$ & 1273,87 & 119,95 \\
\hline 14,00 & $\begin{array}{c}262,9 \\
9\end{array}$ & $\begin{array}{c}251,4 \\
9\end{array}$ & 1301,71 & 83,94 \\
\hline 14,00 & $\begin{array}{c}272,2 \\
4\end{array}$ & $\begin{array}{c}260,7 \\
4\end{array}$ & 1349,59 & 85,40 \\
\hline 21,02 & $\begin{array}{c}164,0 \\
0\end{array}$ & $\begin{array}{c}152,5 \\
0\end{array}$ & 525,70 & 44,15 \\
\hline 21,02 & $\begin{array}{c}255,5 \\
0\end{array}$ & $\begin{array}{c}244,0 \\
0\end{array}$ & 841,13 & 55,10 \\
\hline 28,00 & $\begin{array}{c}117,2 \\
0\end{array}$ & $\begin{array}{c}105,7 \\
0\end{array}$ & 273,55 & 28,02 \\
\hline 28,00 & $\begin{array}{c}269,6 \\
0\end{array}$ & $\begin{array}{c}258,1 \\
0\end{array}$ & 667,96 & 42,49 \\
\hline 35,01 & $\begin{array}{c}190,3 \\
0\end{array}$ & $\begin{array}{c}178,8 \\
0\end{array}$ & 370,08 & 28,55 \\
\hline 35,01 & $\begin{array}{c}129,9 \\
5\end{array}$ & $\begin{array}{c}118,4 \\
5\end{array}$ & 245,17 & 23,59 \\
\hline
\end{tabular}

Fuente: IRD-Brasil

\subsection{Dosimetría ambiental TLD}

La dosimetría ambiental termoluminiscente llevada a cabo en la mina fue comparada con la obtenida en una estación de muestreo ambiental de la ciudad de Arequipa, ubicada aproximadamente a 3000 metros sobre el nivel del mar (Tabla 2).

Tabla 2: Dosimetría ambiental comparativa.

\begin{tabular}{|c|c|}
\hline Estación & $\begin{array}{c}\text { Tasa de exposición } \\
(\boldsymbol{\mu R / h})\end{array}$ \\
\hline Arequipa & $7,2 \pm 0,6$ \\
\hline Mina Coricancha & $11,9 \pm 0,7$ \\
\hline
\end{tabular}

Fuente: LSCD-IPEN

\section{Interpretación de los resultados}

Los resultados de la concentración de radón en aire con detectores de trazas nucleares presentaron mucha variabilidad especialmente en los colectados durante las primeras semanas; esto puede ser 
debido a múltiples factores como por ejemplo las condiciones ambientales tales como la altura, presión y humedad, característicos de una mina ubicada a 4000 metros de altura sobre el nivel del mar y que pueden haber afectado la capacidad de estos detectores. Asimismo, las actividades de la zona, procesos de ventilación y otros pueden generar estas variaciones en la concentración de radón.

Veiga y colaboradores encontraron en minas subterráneas de Brasil niveles de radón con rangos superiores a los encontrados en el presente estudio [5]. Esto se debería a las características geológicas del territorio brasileño.

El Reglamento de Seguridad Radiológica establece en su Anexo IV el nivel de actuación para una acción reparadora en la exposición crónica al radón en puestos de trabajo una concentración media anual de $1000 \mathrm{~Bq} / \mathrm{m}^{3}$ en aire [6]. Considerando que la media de concentración de radón en esta mina es igual a $874,6 \mathrm{~Bq} / \mathrm{m}^{3}$, no se requiere llevar a cabo una acción reparadora en la exposición crónica al radón.

En base a los resultados obtenidos con los dosímetros TLD, se estimó un Equivalente de Dosis Ambiental Hp (10) igual a 0,6 y 0,9 miliSievert/año (mSv/a) para Arequipa y la mina Coricancha respectivamente, si bien en el entorno de la mina la dosis anual es $50 \%$ superior a la de Arequipa, en ambos casos los valores se encuentran por debajo de los 2,4 mSv/a que recibe la población mundial promedio debido a la contribución de radiactividad natural [7].

\section{Conclusiones}

Los niveles de concentración de radón encontrados en la mina Coricancha superan en algunos dispositivos los límites establecidos para una acción reparadora, sin embargo, el valor medio anual se encontraría dentro del rango permisible.

Considerando que los valores dosimétricos anuales se encuentran por debajo del valor medio mundial, el principal problema de exposición a las radiaciones en los trabajadores mineros se deberá a exposición por incorporación interna.

La monitorización de radón en minas subterráneas andinas representa un problema técnico que requiere mejor evaluación dado que los dispositivos de detección que existen en la actualidad son muy sensibles a las condiciones ambientales extremas que existen en nuestro territorio.
Se recomienda ampliar estas investigaciones en zonas mineras ubicadas en áreas geológicas favorables para la explotación de uranio.

\section{Agradecimientos}

Los participantes del proyecto agradecen a la Ing. Marta Ly por su invalorable apoyo logístico para el transporte de nuestro personal y equipos a la zona de trabajo durante el tiempo de ejecución del proyecto. Asimismo, se agradece al Ing. Ernesto Tapia R., Capitán de la mina Coricancha por su apoyo en el mantenimiento y seguridad de los dispositivos de monitoreo, así como el apoyo en las labores de trabajo del personal científico en la mina

\section{Referencias}

[1] E.M. El Afifi, M.A. Hilal, S.M. Khalifa y H.F. Aly, Radiation Measurements. 415 (2006) 627-633.

[2] Mudd, G.M. Rev Environ Sci Biotechnol, 7 (2008) 325

[3] L. Canepa y F. Rosado, Uranium Deposits in Latin America: Geology and Exploration (IAEA-AG-162/16, 1981), pp. 269-300.

[4] M.M. Monnin y J.L. Seidel, Radon Measurements by Etched Track Detectors, (World Scientific Publishing Co., 1997), pp. 51-65.

[5] L.H.S. Veiga, V. Melo, S. Koifman y E.C.S. Amaral, J. Radio. Prot. 24 (2004) 295

[6] Ministerio Energía y Minas, Reglamento de Seguridad Radiológica, Decreto Supremo 009-97-EM.

[7] M. Charles, J. Radio. Prot. 21 (2001) 83

Email: josores@ipen.gob.pe 